\title{
DEVELOPMENT OF NUMERICAL PROCEDURE ON ICE ACCRETION PHENOMENA CONSIDERING SPLASH AND BOUND
}

\author{
K. Isobe ${ }^{1}$, M. Suzuki ${ }^{2}$ and M. Yamamoto ${ }^{3}$ \\ ${ }^{1}$ Graduate school of Mechanical Engineering, Tokyo University of Science \\ ${ }^{2}$ Clean Engine Team, Aviation Program Group, Japan Aerospace Exploration Agency \\ ${ }^{3}$ Department of Mechanical Engineering, Tokyo University of Science \\ (yamamoto@rs.kagu.tus.ac.jp)
}

\begin{abstract}
Ice accretion is a phenomenon that super-cooled water droplets impinge and accrete on a body. It is well known that ice accretion on blades and airfoils leads to aerodynamic performance degradation and has caused severe accidents. The flight test for ice accretion is too expensive and difficult to reproduce every climate condition where ice accretion occurs. Therefore, the numerical simulation is very useful to predict or estimate ice accretion. In the previous researches, various models have been proposed, but the predicted results were in insufficient agreement with experimental and field data. One of the reasons for the disagreement is that the model of SLD(Super-cooled Large Droplet) has not been validated. Therefore, in this study, the authors applied the SLD model to the NACA0012 airfoil and compared the results with the experimental data. As a result, the computations without the model excessively overpredicted the experimental results, but the results with the model provided a closer agreement with the experimental results on the accreted ice shape.
\end{abstract}

Keywords: Computational Fluid Dynamics, Icing, Super-cooled Large Droplet.

\section{INTRODUCTION}

Ice accretion is a phenomenon where super-cooled water droplets impinge and accrete on a body. Ice accretion occurs on airplanes, windmills at cold districts, electric lines and so on. It has caused various accidents, especially in the air transport. One of most notable accidents is Air Florida Flight 90, a Boeing 737 that crashed just after take off in a blizzard on January 13th, 1982. This caused 78 deaths. Another instance of a crash in flight due to ice accretion occurred on October 31st, 1994. This was American Eagle Flight 4184. This ATR72-212, a twin turboprop airliner, was in a holding pattern around Chicago O'Hare International Airport because of weather delays. While holding, it endured freezing rain, a condition where super-cooled water droplets impacted on the wings, causing a rapid ice buildup. This led to lose the control. The most recent accident is Continental Flight 3407, a Bombardier 
DHC8 on February 12th, 2009. It crashed because of using an auto pilot system under icing condition, causing 50 deaths. Although various anti-icing and deicing systems have been developed, these accidents still occur. Therefore, it is important to clarify phenomena of ice accretion. The estimation of ice accretion is necessary to avoid accidents and useful to reduce the cost and the design time in the design phase of aircrafts and jet engines. However, experimental investigations are very difficult, because it is not easy to set ice accretion conditions repeatedly in a wind tunnel and reproduce the various climate conditions. Therefore, it is expected computational fluid dynamics (CFD) which can estimate the ice accretion in the various climate conditions will be a useful way to predict ice accretion phenomenon.

One of the causes of the accidents due to ice accretion is super-cooled large droplets (SLD). In fact, the cause of the accident which happened in 1994 as mentioned above was the ice accretion of SLD. The characteristic phenomena of SLD are splash and bound of impinging droplets. Splash is the phenomenon that a SLD breaks up when it impinges on a surface, and a part of the water mass sticks on the impingement area and the remaining water mass rebounds from the surface. Bound is the phenomenon that a SLD takes a perfect elastic collision at the surface and the water mass is conserved. The ice accretion of a SLD has been modeled by a lot of researchers, but the phenomena and the models have not been validated sufficiently.

In this study, we focus on an airplane, because it is well known that a decline of aerodynamic performance is caused by the deformation of the airfoil shape due to ice accretion. We validate a SLD ice accretion model considering splash and bound phenomena with the Euler-Lagrange coupling and applied the model to a NACA0012 airfoil under several icing conditions. The numerical results are compared with the experimental data to evaluate a validity of the model.

\section{COMPUTATIONAL METHOD}

Flowchart used in this study is shown in Figure 1. At first, we calculate the flow field around a clean wing. Next, we obtain the distribution of droplets impingement on the wing surface by the droplet trajectory calculation. Next, we calculate the ice thickness by the thermodynamic calculation and obtain an ice shape. Finally, the computational grid is remeshed. The procedure is repeated until the termination time of ice accretion is reached.

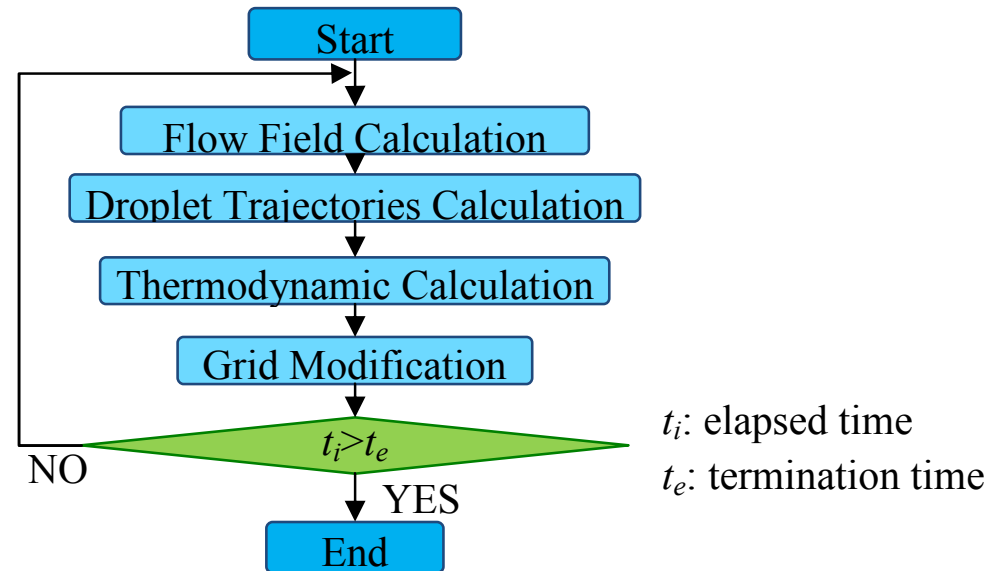

Figure 1 Flow chart of ice accretion simulation 


\subsection{Flow field}

The flow field is assumed to be two-dimensional, compressible and turbulent. The governing equations are Favre-averaged continuity, Navier-Stokes and energy equations. The standard $k-\varepsilon$ turbulence model (Launder and Spalding, [1]) is applied to estimate turbulence. The governing equations are discretized using second-order upwind TVD scheme (Yee and Harten, [2]) for the inviscid terms, second-order central difference scheme for the viscous ones, and 4-stage Runge-Kutta method for the time integration.

\subsection{Droplet trajectories}

Droplet trajectory calculation based on a Lagrangian approach is performed to obtain the droplet collection efficiency on the wing. The calculation uses the following assumptions:

- The droplet is solid and spherical.

- The droplet does not break up.

- The droplets do not interact with each other.

- The droplets do not affect on the flow field (one-way coupling).

- The initial droplet velocity is equal to the gas velocity at the release point.

The equation of droplet motion is

$$
\frac{d \vec{U}_{p}}{d t}=\frac{3}{4} C_{D} \frac{\rho_{f}}{\rho_{p}} \frac{1}{d_{p}} \vec{U}_{r}\left|\vec{U}_{r}\right|
$$

where $t$ is the time, $U_{p}$ is the droplet velocity, $U_{r}$ is the relative velocity between the gas and the droplet, $d_{p}$ is the droplet diameter, and $\rho_{f}$ and $\rho_{p}$ are the gas and the droplet density, respectively. The drag coefficient $C_{D}$ is expressed as:

$$
C_{D}=\frac{24}{\operatorname{Re}_{p}}\left(1+0.15 \operatorname{Re}_{p}^{0.687}\right)
$$

where $R e_{p}$ is the Reynolds number of the droplet based on the diameter and the relative velocity between the gas and the droplet.

\subsection{Thermodynamics}

The model of thermodynamics is the extend Messinger model [3], based on Stefan problem which is generic method of phase change. The governing equations can be written as

$$
\begin{gathered}
\frac{\partial T_{i}}{\partial t}=\frac{k_{i}}{\rho_{i} C_{p i}} \frac{\partial^{2} T_{i}}{\partial y^{2}} \\
\frac{\partial T_{w}}{\partial t}=\frac{k_{w}}{\rho_{w} C_{p w}} \frac{\partial^{2} T_{w}}{\partial y^{2}}
\end{gathered}
$$




$$
\begin{gathered}
\rho_{i} \frac{\partial h_{i}}{\partial t}+\rho_{w} \frac{\partial h_{w}}{\partial t}=m_{i m}+m_{i n}-m_{e, s} \\
\rho_{i} L_{F} \frac{\partial h_{i}}{\partial t}=k_{i} \frac{\partial T_{i}}{\partial y}-k_{w} \frac{\partial T_{w}}{\partial y}
\end{gathered}
$$

where Eqs. (3) and (4) are the energy equations of ice and water layers respectively, Eq. (5) is the mass conservation, Eq. (6) is the condition of phase change at ice/water interface. $\rho, T, k$, $C_{p}$ and $h$ denote the density, temperature, thermal conductivity, specific heat and thickness respectively. The subscript $i$ and $w$ indicate ice and water respectively. $t$ is the time, $y$ is the normal direction to a wall. $m_{i m}, m_{i n}$ and $m_{e, s}$ are the mass flow rate of impinged droplets, runback in and evaporate (sublimation). $L_{F}$ is the latent heat of water freezing. Ice thickness is determined by solving these equations.

\section{MODEL OF SPLASH AND BOUND}

In this study, the SLD ice accretion model proposed by Wright[4] is adopted. This model is a modification of the SLD model proposed by Trujillo [5]. When a droplet impinges on a wing surface, the behavior of droplet (bound, splash and stick) are estimated by Eqs (7) and (8).

$$
\begin{gathered}
K_{L}=\frac{0.859 \times \sqrt{\mathrm{Oh}_{w} \mathrm{Re}_{w}^{5 / 4}}\left(\frac{\rho_{w}}{L W C}\right)^{1 / 8}}{(\sin \alpha)^{5 / 4}} \\
\frac{d_{\text {after }}}{d}=8.72 \times \exp \left(-0.0281 \times \mathrm{Oh}_{w} \mathrm{Re}_{w}^{5 / 4}\right)
\end{gathered}
$$

where $\mathrm{Oh}_{w}=\mu_{w} /\left(\rho_{w} \sigma_{w} d\right)^{1 / 2}, \quad \operatorname{Re}_{w}=d V_{n} \rho_{w} / \mu_{w} . \sigma$ and $\mu$ are the surface tension and viscosity, $d$ is the diameter of a droplet, $V_{n}$ is the droplet velocity normal to the wall, $\alpha$ is the angle of attack against the wing surface. The no-subscript represents the droplet condition before impingement, and the subscript after denotes the droplet condition after impingement. The droplet behavior is judged by the parameter $K_{L}$ in Eq. (7). If $K_{L}$ is lower than 200, the droplet sticks on the impingement area, and if $K_{L}$ is higher than 200, the droplet radii ratio of impingement to rebound (secondary droplet) is calculated by Eq. (8). Next, if radii ratio is lower than 0.05 , the droplet sticks on the impingement area, and if the droplet radii ratio is not less than 0.05 but not more than 1, the droplet splashes, and if the droplet radii ratio is larger than 1, the droplet bounds. When the droplet is judged as splash, the droplet is divided into small secondary droplets which have different diameters. In this study, the only one droplet with a typical diameter calculated by Eq. (8) is traced, but not all secondary droplets are traced.

The mass ratio of the droplet before splash to the secondary droplet is calculated by Eq. (9), the ratios of the tangential velocities and the normal velocities are given by Eqs. (10) and (11). In these equations, $m$ is the mass at the time of impingement, $m_{\text {loss }}$ is the mass loss of 
droplet, $V$ is the droplet velocity, and the subscript $t$ denotes the tangential direction and $n$ denotes the normal direction to the surface.

$$
\begin{gathered}
\frac{m_{\text {loss }}}{m}=0.7(1-\sin \alpha)\left\{1-\exp \left[-0.0092026\left(K_{L}-200\right)\right]\right\} \\
\frac{V_{t, a f t e r}}{V_{t}}=1.075-0.0025 \alpha \\
\frac{V_{n, \text { after }}}{V_{n}}=0.3-0.002 \alpha
\end{gathered}
$$

When the judgment is "splash", the mass loss of droplet is regarded as the mass of secondary droplet and the remaining mass of droplet sticks on the impingement area. When the judgment is "bound", the droplet radii ratio is 1 , and the mass loss of droplet is 0 . When the secondary droplet impinges on the wing surface, the behavior of droplet is judged again.

\section{COMPUTATIONL CONDITION}

The computational target of this study is a NACA0012 airfoil. The numerical results are compared with the experimental data by Anderson et al [6]. We used the overlapping grid system to enhance the accuracy of the computation. We placed a main grid which is large enough to calculate the flow around the wing, and a sub grid nearby the leading edge. Figure 2 shows the enlarged view of these grids around the leading edge. The main grid consists of $161 \times 81$ grid points and the sub grid consists of $121 \times 31$ grid points. The number of the total grid points is about 16,800. Table 1 lists the computational conditions. In this study, we simulated four cases with different droplet diameters, to confirm the effect of droplet diameter. In Table 1, $L W C$ means the liquid water content and $M V D$ is the median volume diameter of droplets.

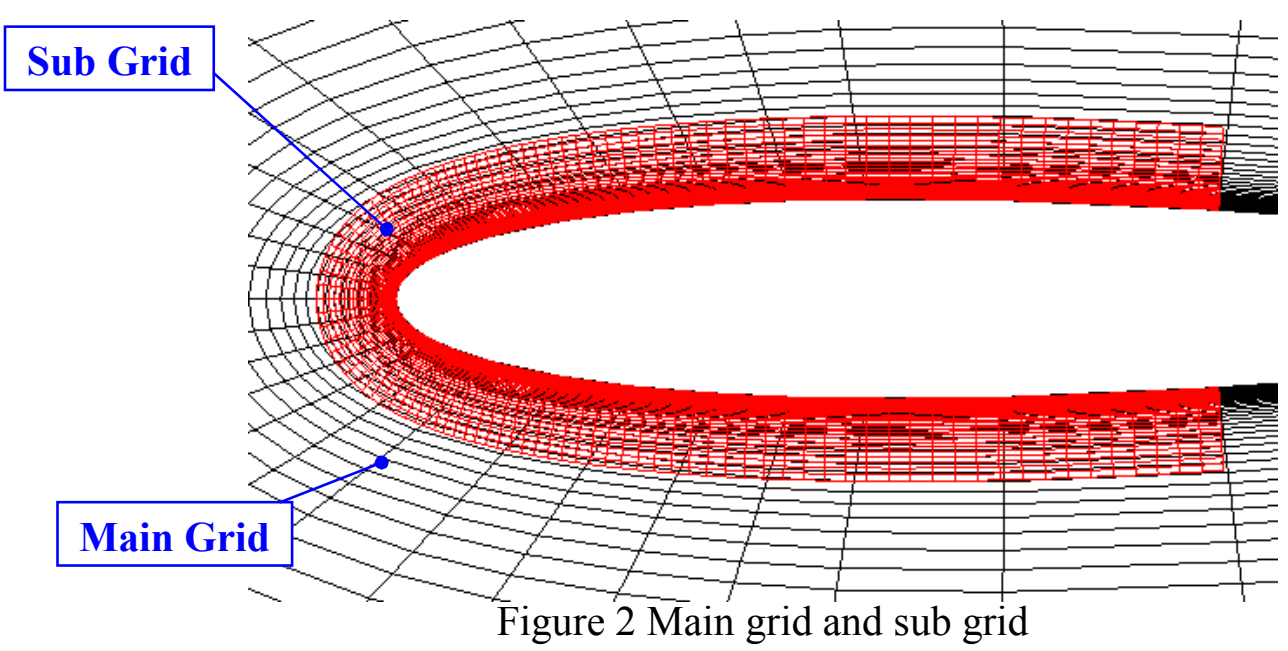


Table 1 Computational condition

\begin{tabular}{|c|c|c|c|c|}
\hline & Case1 & Case2 & Case3 & Case4 \\
\hline $\left.\begin{array}{c}\text { Inflow Static } \\
\text { Temperature }\end{array}{ }^{\circ} \mathrm{C}\right]$ & -8.0 & -8.0 & -10.0 & -6.0 \\
\hline Accretion Time $[\mathrm{s}]$ & 426 & 1110 & 2202 & 1602 \\
\hline Inflow Velocity $[\mathrm{m} / \mathrm{s}]$ & 51.0 & 51.4 & 51.4 & 51.4 \\
\hline MVD $[\mu \mathrm{m}]$ & 200 & 115 & 85 & 30 \\
\hline LWC $\left[\mathrm{g} / \mathrm{m}^{3}\right]$ & 1.2 & 0.90 & 0.96 & 0.84 \\
\hline Chord Length $[\mathrm{m}]$ & 0.533 & 0.914 & 1.829 & 0.914 \\
\hline
\end{tabular}

\section{RESULT AND DISCUSSION}

\subsection{Comparison of the computational result with the experimental results (Case1)}

We compare the results in Case1 with the experimental data [6], because Case1 has the largest diameter and it is the typical case of SLD icing.

\subsubsection{Droplet trajectories and collection efficiency}

The cases considering the SLD model are called as "Modified", and the cases without the SLD model are called as "Normal". Figure 3 shows droplet trajectories before the ice accretion. It indicates that the rebound phenomenon does not occur near the leading edge and occurs a little away from the leading edge. This result is caused from the fact that the parameter $K_{L}$ in Eq. (7) depends on the impact angle strongly.

Figure 4 shows the collection efficiency before the ice accretion. The abscissa $x$ is the distance from the leading edge which is non-dimensionalized by the chord length $c$. The red line illustrates the result of Modified and the green line is the result of Normal. In the result of Normal, the droplets stick on a wider basis. While, in the result of Modified, the collection efficiency decreases because of the effect of the rebound phenomenon.

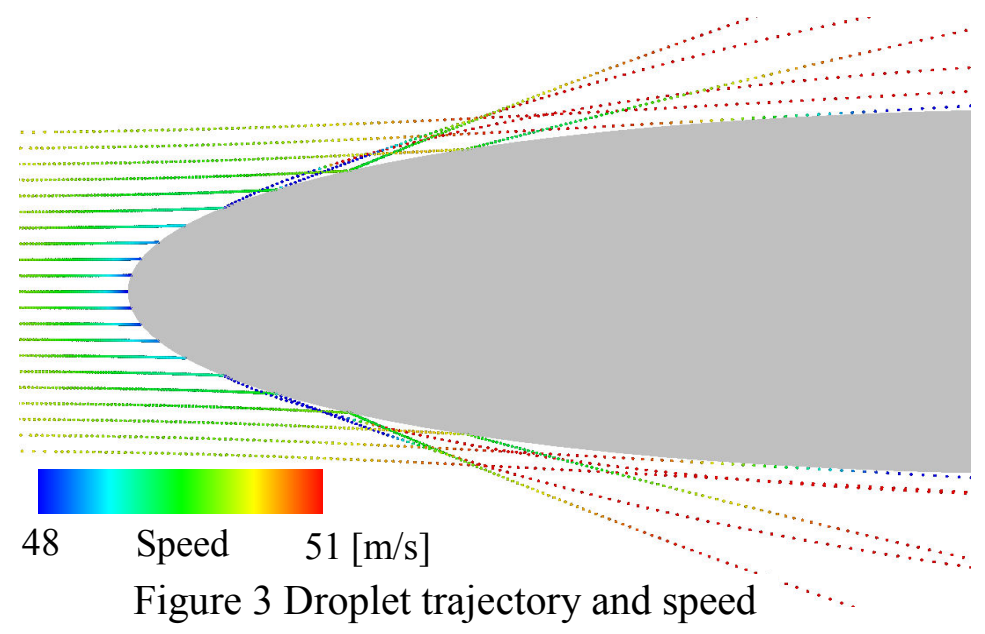




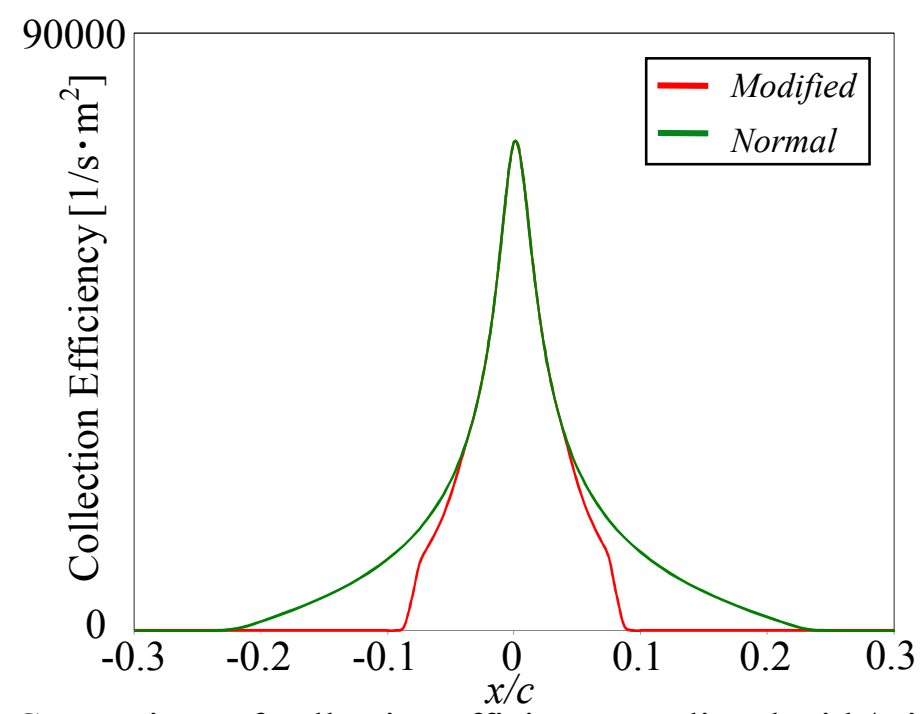

Figure 4 Comparison of collection efficiency predicted with/without SLD model

\subsubsection{Ice shape}

In this chapter, we compare the numerical results with the experimental result on the ice shape. Figure 5 exhibits the ice shapes. The origin is at the leading edge, and $x$ and $y$ coordinate are non-dimensionalized by the chord length $c$. Apparently, The result of Modified is closer to the experimental result than that of Normal. This is caused from the fact that the collection efficiency decreases over the area of $x / c>0.1$ by the effect of splash and bound (see Figure 4). At the leading edge, obviously, the SLD model does not influence on the ice shape. Figure 6 shows the error against the experimental result. The evaluation parameters are ice area, ice accretion limit on the upper and lower surfaces (the wing surface distance from the leading edge to the end of the ice accretion). On the parameter of ice area, the result of Normal is overpredicted by about $50 \%$, while the result of Modified is underpredicted by about less than $10 \%$. The ice accretion limits indicate the similar results. The results of Normal on the upper and lower surfaces are overpredicted by about $100 \%$ and $90 \%$ respectively, while the results of Modified are underpredicted by about less than $10 \%$.

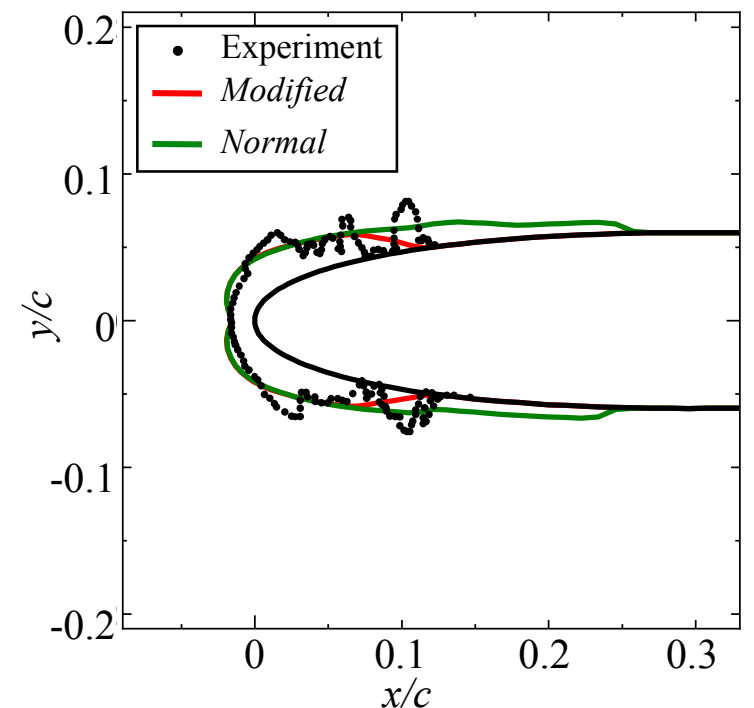

Figure 5 Ice shape (Case1)

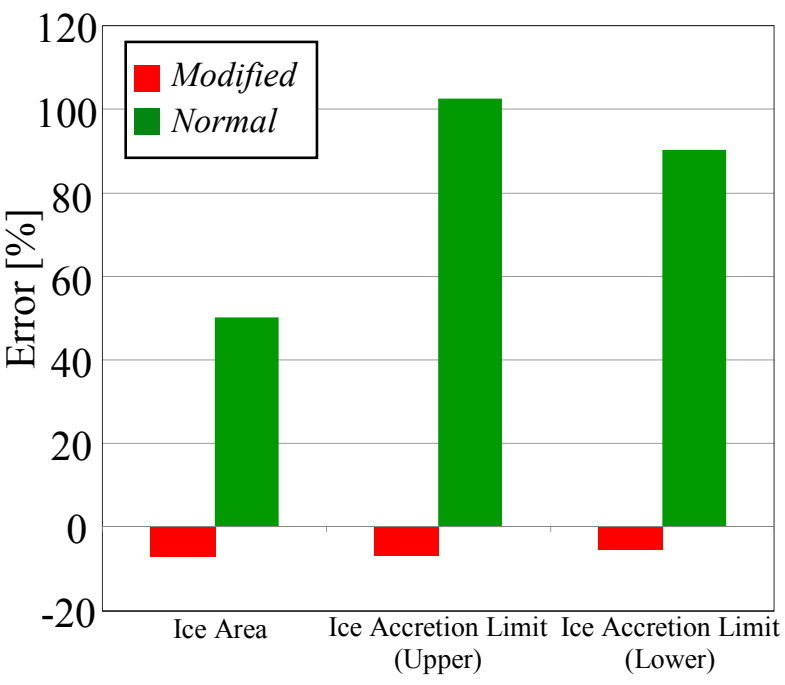

Figure 6 Error of ice shape 


\subsection{Effect of droplets diameter}

In this chapter, we investigate the effect of splash and bound by comparing different cases with different droplet diameters (see Table 1). The ice shape of Case1 is shown in Figure 5 and Case2 4 are shown in Figures 7 9.

From these figures, we can confirm that the smaller diameter of droplet becomes, the weaker influence of the SLD model becomes. There might be two causes. First, the number of droplets which tend to rebound decreases when the droplets are small, because these droplets do not hit the wing surface at low angle. Second, if the small droplets impinge on the wing surface, the droplets do not bound because $K_{L}$ is small. Also, the smaller diameter of droplet becomes, the lower ice accretion limit becomes. It is confirmed that the droplets tend to follow the flow and move to backward of the wing without impingement on the wing surface, because the smaller diameter becomes, the weaker inertia force and the larger drag force to fluid becomes. From these results, the SLD model works well to predict the particular phenomena of SLD.

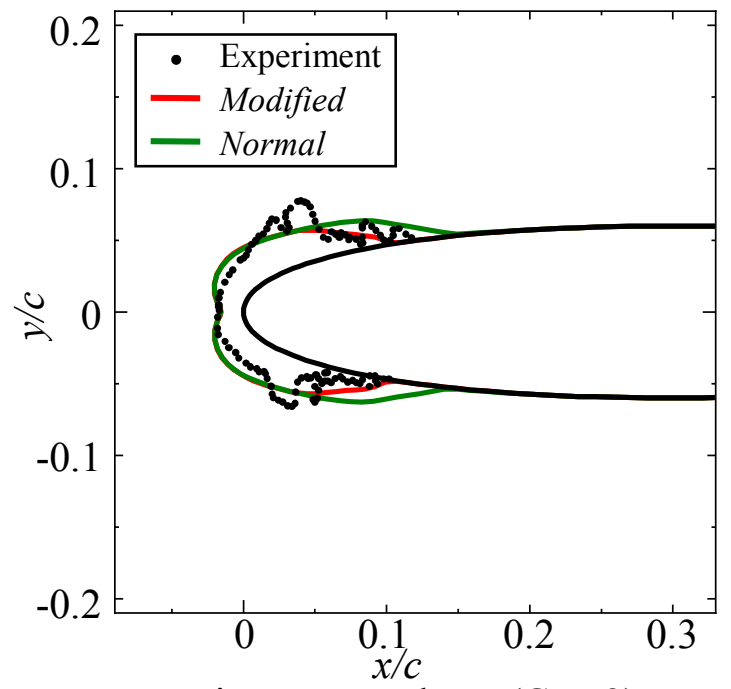

Figure.7 Ice shape (Case2)

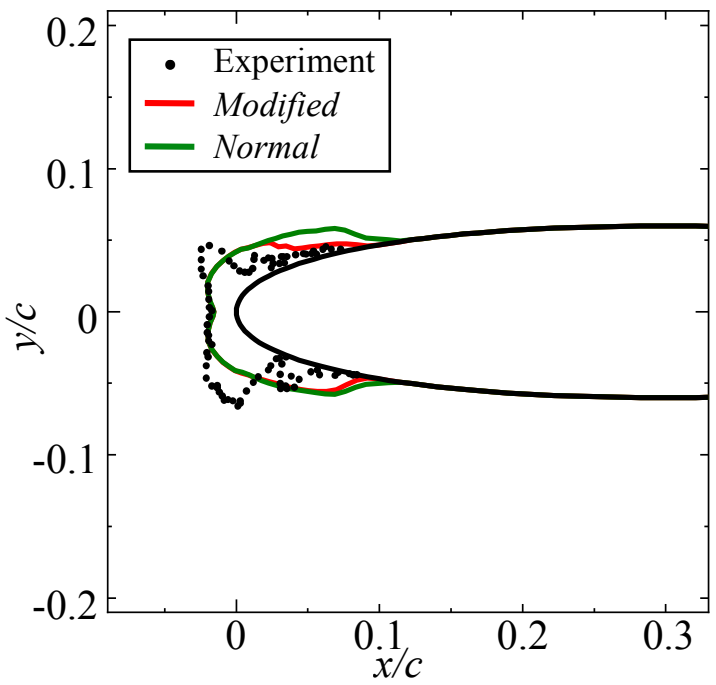

Figure.8 Ice shape (Case3)

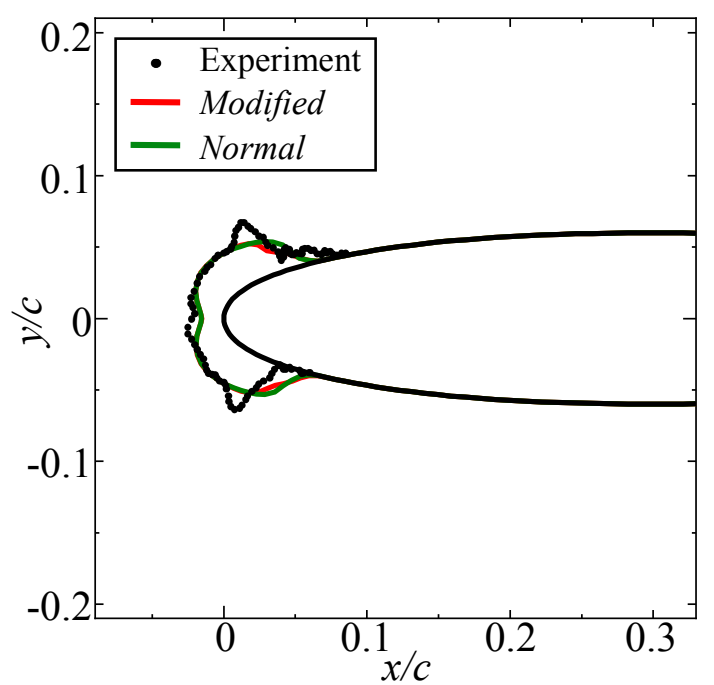

Figure.9 Ice shape (Case4) 


\section{CONCLUSION}

We validated the SLD model in an ice accretion prediction, with considering splash and bound phenomena. The knowledge obtained in the present study is described below:

- On the droplet trajectories, the effect of SLD model is small at the leading edge but is large nearby ice accretion limit, when splash and bound model are included.

- We can decrease overprediction of ice shape by including the SLD model.

- The effect of this model is remarkable in only the SLD condition.

- The smaller droplet becomes, the smaller effect of this model becomes.

In future works, the SLD model should be validated in three-dimensional flow fields.

\section{REFERENCE}

[1] Launder B.E., Spalding D. B., "The Numerical Computation of Turbulent Flows", Computer methods in applied mechanics and engineering, 3, 2, 269-289, 1974.

[2] Yee H. C., "Upwind and Symmetric Shock-capturing Schemes", NASA-TM-89464, 1987.

[3] Ozgen S., Canibek M., "Ice accretion simulation on multi-element airfoils using extended Messinger model", Heat and Mass Transfer, 45, 305-322, 2009.

[4] Wright B.W., Potapczuk, M.G., Levinson L.H., "Comparison of LEWICE and GlennICE in SLD regime", NASA TM 215174, 1-24, 2008.

[5] Trujillo M.F., Mathews W.S., "Modeling and experiment of impingement and atomization of a liquid spray on a wall”, International Journal of Engine, 1.1, 87-105, 1999.

[6] Anderson D.N., Tsao, J.C., "Additional results of ice-accretion scaling at SLD conditions", NASA CR 213850, 1-11, 2005. 